\title{
Transcriptome dynamics along axolotl regenerative development are consistent with an extensive reduction in gene expression heterogeneity in dedifferentiated cells
}

\author{
Carlos Diaz-Castillo ${ }^{\text {Corresp. } 1}$ \\ ${ }^{1}$ Unaffiliated, Irvine, California, USA \\ Corresponding Author: Carlos Diaz-Castillo \\ Email address: crlsdiazcastillo@gmail.com
}

Although in recent years the study of gene expression variation in the absence of genetic or environmental cues or gene expression heterogeneity has intensified considerably, many basic and applied biological fields still remain unaware of how useful the study of gene expression heterogeneity patterns might be for the characterization of biological systems and/or processes. Largely based on the modulator effect chromatin compaction has for gene expression heterogeneity and the extensive changes in chromatin compaction known to occur for specialized cells that are naturally or artificially induced to revert to less specialized states or dedifferentiate, I recently hypothesized that processes that concur with cell dedifferentiation would show an extensive reduction in gene expression heterogeneity. The confirmation of the existence of such trend could be of wide interest because of the biomedical and biotechnological relevance of cell dedifferentiationbased processes, i.e., regenerative development, cancer, human induced pluripotent stem cells, or plant somatic embryogenesis. Here, I report the first empirical evidence consistent with the existence of an extensive reduction in gene expression heterogeneity for processes that concur with cell dedifferentiation by analyzing transcriptome dynamics along forearm regenerative development in Ambystoma mexicanum or axolotl. Also, I briefly discuss on the utility of the study of gene expression heterogeneity dynamics might have for the characterization of cell dedifferentiation-based processes, and the engineering of tools that afforded better monitoring and modulating such processes. Finally, I reflect on how a transitional reduction in gene expression heterogeneity for dedifferentiated cells can promote a long-term increase in phenotypic heterogeneity following cell dedifferentiation with potential adverse effects for biomedical and biotechnological applications. 
1 Transcriptome dynamics along axolotl regenerative development are consistent with an

2 extensive reduction in gene expression heterogeneity in dedifferentiated cells

3

4

5 Carlos Diaz-Castillo

6301 Gabrielino Drive

7 Irvine, CA, USA

8 crlsdiazcastillo@gmail.com

9 http://crlsdiazcastillo.wixsite.com/molcellbiodiversity 


\section{ABSTRACT}

11 Although in recent years the study of gene expression variation in the absence of genetic or

12 environmental cues or gene expression heterogeneity has intensified considerably, many basic

13 and applied biological fields still remain unaware of how useful the study of gene expression

14 heterogeneity patterns might be for the characterization of biological systems and/or processes.

15 Largely based on the modulator effect chromatin compaction has for gene expression

16 heterogeneity and the extensive changes in chromatin compaction known to occur for specialized

17 cells that are naturally or artificially induced to revert to less specialized states or dedifferentiate,

18 I recently hypothesized that processes that concur with cell dedifferentiation would show an

19 extensive reduction in gene expression heterogeneity. The confirmation of the existence of such

20 trend could be of wide interest because of the biomedical and biotechnological relevance of cell

21 dedifferentiation-based processes, i.e., regenerative development, cancer, human induced

22 pluripotent stem cells, or plant somatic embryogenesis. Here, I report the first empirical evidence

23 consistent with the existence of an extensive reduction in gene expression heterogeneity for

24 processes that concur with cell dedifferentiation by analyzing transcriptome dynamics along

25 forearm regenerative development in Ambystoma mexicanum or axolotl. Also, I briefly discuss

26 on the utility of the study of gene expression heterogeneity dynamics might have for the

27 characterization of cell dedifferentiation-based processes, and the engineering of tools that

28 afforded better monitoring and modulating such processes. Finally, I reflect on how a transitional

29 reduction in gene expression heterogeneity for dedifferentiated cells can promote a long-term

30 increase in phenotypic heterogeneity following cell dedifferentiation with potential adverse

31 effects for biomedical and biotechnological applications. 


\section{INTRODUCTION}

33 One of the most intriguing aspects of biological systems is that they are prone to vary even in the

34 absence of genetic or environmental cues (Ackermann 2015; Altschuler \& Wu 2010; Komin \&

35 Skupin 2017; Liu et al. 2016; Symmons \& Raj 2016). Non-genetic non-environmental

36 phenotypic heterogeneity is dependent on the inherent stochasticity of the physicochemical

37 substrate of biological systems, but also on emergent properties of biological processes like: (i)

38 the frequent dependence of biological processes on low numbers of intervening elements, (ii) the

39 crowded conditions of intracellular compartments, (iii) the fidelity decrease in the multistep

40 transference of information to de novo synthesize active genetic products, (iv) the pulsating

41 nature of RNA transcription, or, (v) the slow dynamics of highly compacted chromatin

42 remodeling needed to grant DNA accessibility to the transcription machinery (Ackermann 2015;

43 Altschuler \& Wu 2010; Komin \& Skupin 2017; Liu et al. 2016; Rivas \& Minton 2016; Symmons

44 \& Raj 2016; Yanagida et al. 2015). Since some of these factors can vary within single cells

45 temporally or in response to environmental cues, between cells within single multicellular

46 organisms, or between unicellular or multicellular organisms within single populations, it would

47 be expected that non-genetic non-environmental phenotypic heterogeneity was itself variable

48 (Ackermann 2015; Altschuler \& Wu 2010; Komin \& Skupin 2017; Liu et al. 2016; Rivas \&

49 Minton 2016; Symmons \& Raj 2016; Yanagida et al. 2015). Thus, the study of variation patterns

50 for non-genetic non-environmental heterogeneities is of potential interest for the characterization

51 of biological properties that promoted such heterogeneities.

52

53 Recently, Diaz-Castillo proposed that processes that concur with cell dedifferentiation would

54 show an extensive reduction in gene expression heterogeneity (Díaz-Castillo 2017b), i.e., the 
55 variation in gene expression detected between cells or organisms with the same genotype when

56 assayed in the same environmental conditions. Cell dedifferentiation refers to cases in which

57 well-differentiated, specialized, non-proliferative cells revert to states characterized by less

58 specialization, the ability of re-differentiating towards different cellular fates, and/or proliferation

59 (Li \& Belmonte 2017; Merrell \& Stanger 2016; Sugiyama 2015; Yamada et al. 2014). Cell

60 dedifferentiation is known to occur in the initial stages of developmental programs activated in

61 response to injury or regenerative development in vertebrates, the formation of masses of

62 undifferentiated cells such as tumors in animals or calli in plants, or the artificial induction of

63 somatic embryogenesis in plants and human pluripotent stem cells for biomedical applications

64 (Li \& Belmonte 2017; Merrell \& Stanger 2016; Sugiyama 2015; Yamada et al. 2014).

65

66 The proposal that an extensive decrease in gene expression heterogeneity is characteristic of

67 processes based on cell dedifferentiation relies on cell dedifferentiation itself being a case of cell

68 convergence, and on dedifferentiating cells tendency to show an extensive relaxation of

69 chromatin (Díaz-Castillo 2017b). Since cell dedifferentiation represents the reversal of cell

70 specialization, it would be expected that dedifferentiated cells were more similar between them

71 than the specialized cells they originated from are. Indeed, the use of information theory to

72 characterize transcriptomes for diverse types of human and murine tumors and the organs they

73 originated from showed not only that cancer transcriptomes become less specialized and more

74 similar among themselves than the transcriptomes of their original organs are, but also that they

75 become very similar to the transcriptomes of undifferentiated embryonic stem cells (Martinez \&

76 Reyes-Valdes 2008; Martinez et al. 2010). 
78 On the other hand, based on the analysis of chromatin markers and the activation of genetic

79 elements usually silent because of their location in chromosome regions with highly compacted

80 chromatin, it has been suggested that both naturally-occurring and artificially-induced cell

81 dedifferentiation is characterized by an extensive relaxation of chromatin throughout the nucleus

82 (El-Badawy \& El-Badri 2015; Feher 2015; Grafi \& Barak 2015; Jiang et al. 2013; Krause et al.

83 2015; Lee et al. 2015; Macia et al. 2015; Sosnik et al. 2017; Wang \& Wang 2012; Zhu et al.

84 2012a). Ultimately, chromosome regions with high chromatin compaction are supposed to

85 promote gene expression heterogeneity because of the slow dynamics of the chromatin

86 remodeling needed to grant DNA accessibility to the transcription machinery (Liu et al. 2016;

87 Symmons \& Raj 2016). The modulating effect chromatin compaction has on gene expression

88 heterogeneity is best exemplified by the phenomenon known as position effect variegation

89 (PEV), and the effect of chromatin compaction modifiers on gene expression and phenotypic

90 heterogeneities. Originally discovered in Drosophila melanogaster, PEV refers to the stochastic

91 variation in expression for genes located close to or embedded within chromosome regions with

92 highly compacted chromatin or heterochromatin (Elgin \& Reuter 2013). Factors that directly or

93 indirectly alter chromatin compaction such as temperature, genetic variation in heterochromatin-

94 forming elements, paternal/maternal chromosome inheritance, or the genomic content in junk

95 DNA have been shown to modulate gene expression heterogeneity itself and the phenotypic

96 manifestation of such heterogeneity as PEV (Díaz-Castillo 2015; Elgin \& Reuter 2013; Maggert

$97 \&$ Golic 2002). Thus, considering that chromatin compaction promotes gene expression

98 heterogeneity, it could be inferred that the extensive reduction in chromatin compaction in

99 dedifferentiating nuclei would cause a reduction of gene expression heterogeneity for many

100 genes throughout the genome. 
102 Because no formal proof exists yet for the extensive reduction in gene expression heterogeneity

103 in dedifferentiating cells, I aimed to find preliminary supporting evidence by focusing on the

104 study of gene expression dynamics along regenerative development in Ambystoma mexicanum or

105 axolotl. First, although not yet completely understood, cell dedifferentiation associated to axolotl

106 regenerative development in response to injury has been and still is an important subject of study

107 (McCusker et al. 2015a). Second, transcriptomic analyses of regenerative development in axolotl

108 constitute an ideal model to study gene expression dynamics for processes that concur with cell

109 dedifferentiation because it would permit studying naturally occurring cell dedifferentiation-

110 based phenomena from the moment they are elicited until their completion. Finally, recent years

111 have seen an increase in transcriptomic studies for axolotl regenerative development models

112 (Bryant et al. 2017; Eo et al. 2012; Gearhart et al. 2015; King \& Yin 2016; Knapp et al. 2013;

113 McCusker et al. 2015b; Monaghan et al. 2012; Monaghan et al. 2009; Pai et al. 2016;

114 Ponomareva et al. 2015; Sabin et al. 2015; Seifert et al. 2012; Sousounis et al. 2014; Stewart et

115 al. 2013; Voss et al. 2015a; Wu et al. 2013). Here, I argue about the adequacy of using one these

116 studies to test the prediction for gene expression heterogeneity dynamics associated to cell

117 dedifferentiation because of its temporal design and remarkable replication level. The results of

118 reanalyzing the chosen dataset are consistent with the possibility that processes that concur with

119 cell dedifferentiation might be characterized by an extensive reduction in gene expression

120 heterogeneity (Díaz-Castillo 2017b). In addition, I briefly reflect on the usefulness of the study

121 of gene expression heterogeneity patterns associated to cell dedifferentiation for biomedical and

122 biotechnological applications.

123 


\section{MATERIALS AND METHODS}

125 Data from the transcriptome study for axolotl regenerative development with the largest

126 biological replication was used to assess if lower levels of biological replication, 3-6 replicates,

127 could difficult the study of gene expression heterogeneity patterns along axolotl regenerative

128 development (Bryant et al. 2017; Eo et al. 2012; Gearhart et al. 2015; King \& Yin 2016; Knapp

129 et al. 2013; McCusker et al. 2015b; Monaghan et al. 2012; Monaghan et al. 2009; Pai et al. 2016;

130 Ponomareva et al. 2015; Sabin et al. 2015; Seifert et al. 2012; Sousounis et al. 2014; Stewart et

131 al. 2013; Voss et al. 2015a; Wu et al. 2013). In 2015, Voss and coworkers inspected gene

132 expression dynamics for 9-10 biological replicates at 20 timepoints along 28 days following

133 axolotl forearm amputation using custom Affymetrix GeneChips that include 20,080 probesets

134 and RNA samples obtained from $1 \mathrm{~mm}$ of heterogeneous tissue from the tips of amputated

135 forearms (Voss et al. 2015a). The dataset including normalized transcript abundance for this

136 study, Voss dataset hereinafter, was obtained from Gene Expression Omnibus database

137 (GSE67118)(Barrett et al. 2013; Voss et al. 2015a). Timepoint, biological replicate, and probeset

138 identifiers from the original study were maintained here (Voss et al. 2015a).

139

140 For each probeset and timepoint, transcript abundance mean and coefficient of variation (CV)

141 were calculated and used as proxies for gene expression level and heterogeneity respectively

142 (Dataset S1). Next, for each probeset and timepoint, transcript abundance and CV using 3, 4, 5,

143 or 6 biological replicates chosen at random were calculated to simulate the effect lower

144 replication would have for gene expression measures reproducibility. The similarity of transcript

145 abundance mean and CV for all probesets in each timepoint in Voss dataset when calculated

146 using all biological replicates and each lower-replication simulation was inspected using Pearson 
$147 r$. Lower-replication random simulations were repeated 1,000 times. Table S1 summarizes

148 Pearson $r$ for each timepoint, while Figure S1 represents Pearson $r$ calculated for three

149 timepoints chosen at random. Increasing biological replication results in a similarity

150 improvement for both transcript abundance measures when compared to measures calculated

151 using all biological replicates. However, the similarity of gene expression measures using lower-

152 replication simulations and all biological replicates is always considerably worse for transcript

153 abundance CV than for transcript abundance mean. Thus, although low biological replication can

154 still be appropriate to study changes in the level of gene expression in response to natural or

155 experimental variables, the study of gene expression heterogeneity dynamics warrants the use of

156 designs with abundant biological replication. For this reason, in subsequent analyses I proceed to

157 use only Voss dataset, which ensures the largest biological replication level for transcriptomic

158 studies of axolotl regenerative development.

160 The Monte Carlo-Wilcoxon matched-pairs signed-ranks test (MCW test hereinafter) was used to study

161 gene expression temporal dynamics along the regenerative development of axolotl forearm using Voss

162 dataset as reference (Díaz-Castillo 2015; Voss et al. 2015a). In essence, MCW is based on the Wilcoxon

163 matched-pairs signed-ranks test which permits assessing if matched pairs of quantitative data sampled in

164 two different conditions tend to be biased in any particular direction (Wilcoxon 1945). This test proceeds

165 by calculating a sum of signed ranks assigned to the elements in the dataset in question in virtue of the

166 subtraction of their matched data. The sum of signed ranks (W) is sensitive both to the number of

167 elements with data biased in one or the other direction, and the extent of these biases. The MCW test

168 variation evaluates the significance of the observed bias for the dataset under study by comparing $\mathrm{W}$

169 calculated before and after randomly rearranging data. MCW is very versatile because data can be

170 randomly rearranged with no restriction or respecting certain aspects of the internal structure of the 
171 dataset to simulate the effect different factors might have on the variation of the measure under study.

172 Here, MCW tests were used to asses if transcript abundance CV was generally lower in post-amputational 173 timepoints (TX) than in the day of the amputation (T0), TX vs T0 comparisons hereinafter. For each TX

174 vs T0 comparison, I calculated a gene expression bias index using transcript abundance CV for each

175 probeset in the dataset (cvGEBI). The main steps for the calculation of cvGEBI are represented in Figure

176 S2. First, for each probeset in the dataset, I subtracted transcript abundance CV for the post-amputational

177 timepoint from transcript abundance $\mathrm{CV}$ for the day of the amputation $\left(\Delta \mathrm{CV}=\mathrm{CV}_{\mathrm{TX}}-\mathrm{CV}_{\mathrm{T} 0}\right)$. Second,

178 probesets were ranked after sorting them using the absolute value of $\Delta \mathrm{CV}$ from smallest to largest. Third,

179 ranks for each probeset were signed using the sign of $\Delta \mathrm{CV}$. Fourth, the sum of signed ranks (W) was

180 calculated as $\sum\left[\operatorname{sgn}\left(\Delta \mathrm{CV}_{\mathrm{i}}\right) \cdot \operatorname{rk}\left(\left|\Delta \mathrm{CV}_{\mathrm{i}}\right|\right)\right]$. Fifth, cvGEBI was calculated as $\mathrm{W} / \mathrm{W}_{\max }$, where $\mathrm{W}_{\max }$ represents

181 the maximum value $\mathrm{W}$ could take. cvGEBIs will range from 1 to -1 if transcript abundance $\mathrm{CV}$ was

182 higher or lower post-amputationally than in the day of the amputation for all the probesets in the dataset, 183 respectively.

185 MCW tests evaluate the significance of observed cvGEBIs by comparing them with simulated cvGEBIs 186 calculated after randomly rearranging transcript abundance CV. Here, three different MCW test designs 187 were used to simulate the effect chance, factors acting on the transcriptome as a whole, or the variation in 188 the mean level of expression would have on transcript abundance CV dynamics along axolotl forearm 189 regenerative development. These three MCW designs are referred to as unrestricted, timepoint-restricted, 190 and expression-restricted MCW tests (Figure S2, and Table S2). Unrestricted MCW tests proceed by 191 recalculating cvGEBIs after randomly rearranging transcript abundance CV with no other restriction.

192 Timepoint-restricted MCWs tests proceed by recalculating cvGEBIs after randomly rearranging transcript

193 abundance CV within each timepoint for each TX vs T0 comparison. Expression-restricted MCW tests

194 proceed by recalculating cvGEBIs after randomly rearranging transcript abundance CV within bins of

195 probesets defined by their corresponding transcript abundance mean values. Probeset bins were defined

196 by rounding up transcript abundance mean values to 2 or 3 decimal digits independently. These two 
197 alternatives differ in the numbers of bins and number of probesets per bin (Table S3). Random

198 permutations of transcript abundance CV for each MCW test were repeated 10,000 times. The

199 significance of observed cvGEBIs was estimated by calculating $P_{\text {upper }}$ and $P_{\text {lower }}$ values as the fraction of

200 simulated cvGEBIs that were higher or equal, or lower or equal than observed cvGEBIs, respectively.

201 Observed cvGEBIs were considered significant if $P_{\text {upper }}$ and $P_{\text {lower }}$ values were lower than 0.05 .

202

203 The proportional cumulative area under the curve $\left(\mathrm{PC}_{\mathrm{AUC}}\right)$ was used to estimate how much of the

204 temporal dynamics for gene expression heterogeneity along the regenerative development of axolotl

205 forearm could be accounted exclusively by chance, factors acting on the transcriptome as a whole, or the

206 variation in the mean level of expression for each probeset (Table $\mathrm{S} 4$ ). $\mathrm{PC}_{\mathrm{AUC}}$ was calculated as the sum

207 of simulated cvGEBIs with the closets value to observed cvGEBIs for the corresponding TX $v s$ T0

208 comparison and all precedent ones divided by the sum of observed cvGEBIs for the corresponding TX $v s$

209 T0 comparison and all precedent ones for unrestricted, timepoint-restricted and expression-restricted

210 MCW tests.

211

212 Unrestricted MCW tests were also used to study the dynamics of the mean level of gene expression along

213 axolotl forearm regenerative development (Table S2). In this case, gene expression bias indexes for each

214 TX vs T0 comparison were calculated following the process previously described but using transcript

215 abundance mean for each probeset and timepoint, and referred to as mGEBIs. The statistical significance

216 of observed mGEBIs was inspected as previously described for cvGEBIs.

217

218 To functionally characterize those genes that contribute to the sharp decrease in gene expression

219 heterogeneity early in the regenerative development of axolotl forearm, gene expression heterogeneity

220 fold change between 1 and 1.5 days post-amputation was calculated for each probeset in the dataset as

$221 \log _{2}\left(\mathrm{CV}_{\mathrm{T} 1.5} / \mathrm{CV}_{\mathrm{T} 1}\right)$, where, $\mathrm{CV}_{\mathrm{T} 1}$ and $\mathrm{CVT}_{1.5}$ correspond to transcript abundance $\mathrm{CV}$ calculated for $\mathrm{T} 1$ and

222 T1.5 timepoints, respectively. In an attempt to distinguish probesets for which gene expression 
223 heterogeneity dynamics between 1 and 1.5 days post-amputation constituted a clear tendency change from

224 those that showed erratic fluctuations within a longer timeline, transcript abundance CV data for 0.5 and 2

225 days post-amputation were also taken into consideration. For those probesets for which transcript

226 abundance $\mathrm{CV}$ was not higher or lower for both 0.5 and 1 days timepoints than for both 1.5 and 2 days

227 timepoints, their $\log _{2}\left(\mathrm{CV}_{\mathrm{T} 1.5} / \mathrm{CV}_{\mathrm{T} 1}\right)$ was set to 0 . GOrilla and REVIGO were used to identify significant

228 enrichments for Gene Ontology (GO) terms (Eden et al. 2009; Supek et al. 2011). GOrilla permits

229 detecting GO term enrichments for genes in the top of a ranked list when compared with the rest of the

230 list using a minimum hypergeometric (mHG) test $\{$ Eden, $2009 \# 41\}$. GOrilla was used to find GO

231 enrichments for all probesets in the dataset ranked using the increasing value of corrected

$232 \log _{2}\left(\mathrm{CV}_{\mathrm{T} 1.5} / \mathrm{CV}_{\mathrm{T1}}\right)$, and 10 lists including all probesets in the dataset randomly rearranged setting the

233 reference GO annotation to Homo sapiens, the running mode to "single ranked list of genes", and, the $P$

234 value threshold to $10^{-3}$ (Table S5). The GO term enrichment found using randomly rearranged probesets

235 supported by the largest number of genes was used as threshold to narrow down observed GO

236 enrichments with potential biological significance. REVIGO was used to minimize GO term redundancies

237 using $P$ values obtained using GOrilla as GO enrichments, and setting allowed similarity to medium, the

238 database with GO term sizes to whole UniProt, and, the semantic similarity to SimRel.

240 The MCW test was also used to study gene expression dynamics for specific subsets of genes defined by

241 their similar functionality when compared with the whole transcriptome along axolotl forearm

242 regenerative development (Figure S2, and Table S2). This new MCW variation is referred to as

243 functionally-restricted MCW test (Figure S2). First, genes classified in human gene ontology classes

244 mitotic cell cycle process (GO:1903047), and chromatin organization (GO:0006325) were retrieved from

245 the Gene Ontology Consortium database (The Gene Ontology Consortium 2015). Functionally-restricted

246 MCWs tests proceed by calculating mGEBIs and cvGEBIs for groups of probesets corresponding to

247 genes classified in each GO class before and after randomly rearranging functional subset tags for the 
248 whole dataset and each TX vs T0 comparison 10,000 times. The statistical significance of observed

249 mGEBIs and cvGEBIs using functionally-restricted MCW tests was inspected as previously described.

250

251 RESULTS

252 An extensive reduction in gene expression heterogeneity is detectable early after axolotl

253 forearm amputation. The axolotl has been a preferred model system for the study of post-

254 traumatic regeneration of complex structures for more than a century (Voss et al. 2015b). During

255 this time, the progress of regenerative development responses has been intensively characterized

256 macroscopically and microscopically (McCusker et al. 2015a). Briefly, immediately after a gross

257 insult such as the amputation of a limb, a number of processes are activated in response to the

258 injury to heal the open wound (McCusker et al. 2015a). Shortly after that, the amputation plane

259 gets populated with regeneration-competent progenitor cells, collectively referred to as the

260 blastema, which will coordinately grow and pattern to restore the missing structure (McCusker et

261 al. 2015a). A long-held view assumes that dedifferentiating cells are the main contributor to the

262 formation of the blastema, and evidence of cell dedifferentiation upon injury have been found for

263 different cell types such as muscle cells, keratinocytes or fibroblasts (McCusker et al. 2015a).

264 However, recent lineage-tracing analyses raised some doubts on how many cell types actually

265 dedifferentiate in response to injury, and for those that do, how they contribute to the

266 regeneration of the different tissues needed to restore the missing structure (Kragl et al. 2009;

267 Sandoval-Guzman et al. 2014; Wu et al. 2015). Conversely, the study of heterogeneous samples

268 from post-amputation axolotl limb blastemas have shown the activation shortly after amputation

269 of genes commonly expressed in germ line cells, and transposable elements (TE) commonly

270 silent because of their location in regions with highly compacted chromatin (Zhu et al. 2012a;

271 Zhu et al. 2012b). Since TE activation is known to occur as a consequence of the extensive 
272 chromatin relaxation in un/dedifferentiated cells (Feher 2015; Grafi \& Barak 2015; Macia et al.

273 2015; Wang \& Wang 2012), TE activations detected in axolotl regenerative development

274 responses might be consistent with the possibility that the formation of the blastema required, at

275 least partially, dedifferentiating cells that undergo extensive chromatin relaxation. Furthermore,

276 Sosnik et al. recently developed a new method to quantify the nuclear condensation using

277 microscopic images and demonstrated that blastemal cells show a significant reduction in

278 chromatin condensation when compared with somatic dermal cells (Sosnik et al. 2017). Thus, the

279 use of transcriptomic analyses for axolotl regenerative development models seems like an

280 adequate place to seek preliminary support for the hypothesized extensive decrease in gene

281 expression heterogeneity for processes that concur with cell dedifferentiation (Díaz-Castillo 282 2017b).

283

284 In recent years, the number of studies of transcriptome dynamics for axolotl regenerative 285 development models increased considerably (Bryant et al. 2017; Eo et al. 2012; Gearhart et al. 286 2015; King \& Yin 2016; Knapp et al. 2013; McCusker et al. 2015b; Monaghan et al. 2012;

287 Monaghan et al. 2009; Pai et al. 2016; Ponomareva et al. 2015; Sabin et al. 2015; Seifert et al.

288 2012; Sousounis et al. 2014; Stewart et al. 2013; Voss et al. 2015a; Wu et al. 2013). A common 289 aspect to many of these studies is that they encompass very low levels of biological replication, 290 i.e., 1-6 replicates per condition, which can constitute a big obstacle for the study of gene 291 expression heterogeneity dynamics (see Materials and Methods, Figure S1, and Table S1). For 292 this reason, all analyses presented here used the dataset produced by the transcriptome study for 293 axolotl regenerative development with the largest biological replication level. In 2015, Voss and 294 coworkers analyzed transcriptome dynamics during the initial 28 days of axolotl post-amputation 
295 forearm regenerative development using $1 \mathrm{~mm}$ of heterogeneous tissue from the tip of amputated

296 and post-amputational regenerating forearms at 19 timepoints (Voss et al. 2015a). More

297 importantly, the study performed by Voss and coworkers consisted of 9-10 biological replicates

298 per timepoint for a region of post-amputational regenerating forearms where the blastema is

299 formed and maintained along the regenerative process (Voss et al. 2015a).

300

301 To characterize gene expression heterogeneity dynamics along axolotl forearm regenerative

302 development, transcript abundance coefficient of variation (CV) was used as a measure of gene

303 expression heterogeneity for each probeset and timepoint in Voss dataset. Also, a variation of the

304 Monte Carlo-Wilcoxon matched-pairs signed-ranks test (MCW test hereinafter) was used to

305 calculate the gene expression bias index (GEBI) that quantifies the bias for gene expression

306 measures for a group of genes between two conditions and test their statistical relevance by

307 comparing them with GEBIs calculated after randomly rearranging these measures (Figure S2)

308 (Díaz-Castillo 2015). MCW test is particularly suited to study the existence of an extensive bias

309 for a given quantitative measure between two conditions because it is sensitive to the number of

310 elements within the studied dataset with biased measures and the extent of such biases, but also

311 because it considers all the elements in the studied dataset instead of only those that are deemed

312 significant in virtue of arbitrary thresholds (Díaz-Castillo 2015). For this particular case, MCW

313 test was used to confirm the existence of gene expression heterogeneity biases between each one

314 of the post-amputational timepoints (TX) and the day of the amputation (T0), TX vs T0 pairwise

315 comparisons. cvGEBIs, indexes calculated using transcript abundance $\mathrm{CV}$, range from 1 if for all

316 probesets under study transcript abundance CV was larger post-amputationally than in the day of

317 the amputation $\left(\mathrm{CV}_{\mathrm{TX}}>\mathrm{CV}_{\mathrm{T} 0}\right)$, and -1 if for all probesets under study transcript abundance $\mathrm{CV}$ 
318 was lower post-amputationally than in the day of the amputation $\left(\mathrm{CV}_{\mathrm{TX}}<\mathrm{CV}_{\mathrm{T} 0}\right)$. To ascertain if

319 observed gene expression heterogeneity biases for each TX vs T0 comparison were significantly

320 different from those expected for pairwise comparisons with the same number of elements and

321 value distribution just by chance, cvGEBIs were recalculated after randomly rearranging

322 transcript abundance CV 10,000 times with no restriction. Since later on MCW tests with

323 different designs will be used to address different proximate questions, the design with

324 unrestricted permutation of gene expression measures will be referred to as unrestricted MCW

325 tests.

326

327 Blastemas typically form 6-12 days after amputation depending on the animal size and age (Voss

328 et al. 2015a). If the blastema formation indeed encompassed intervening cell dedifferentiation,

329 and this concurred with an extensive relaxation of chromatin, we would expect that cvGEBIs

330 became negative and significantly different from those expected by chance from day 6-12

331 onwards. Somehow surprisingly, not only cvGEBIs were significantly different for the expected

332 timepoints, but they started being significantly negative as early as 1.5 days after amputation

333 (Figure 1, and Table S2).

334

335 An intriguing aspect of gene expression heterogeneity dynamics along the regenerative

336 development of axolotl forearm is that it is considerably variable itself (Figure 1, and Table S2).

337 Interestingly, cvGEBI variation is reminiscent of the progression of axolotl forearm regenerative

338 development stages defined morphologically (Voss et al. 2015a). In general, while significantly

339 negative cvGEBIs tend to be higher for timepoints that correspond to the transition between

340 regenerative development stages, and lower for intermediate timepoints for each stage (Figure 1, 
341 and Table S2). For example, an increase in cvGEBIs can be observed in the transition between

342 pre-bud and early bud stages, or medium and late bud stages, whereas the lowest cvGEBIs

343 correspond to intermediate timepoints for medium bud and pallet stages (Figure 1, and Table

344 S2).

345

346 Voss and coworkers documented that samples corresponding to the same timepoint can be

347 classified into consecutive morphological stages, and that this staging imprecision is more

348 accentuated for timepoints corresponding to the transition between stages than for intermediate

349 timepoints within each stage (Voss et al. 2015a). An accentuated variation in the developmental

350 progression of axolotl regenerative development is well known even under highly controlled

351 conditions (Tank et al. 1976). Thus, the parallelism observed for cvGEBIs dynamics here and

352 morphological changes in the original study for axolotl forearm regenerative development

353 suggests that the methodology used here to analyze gene expression heterogeneity dynamics is

354 sensitive enough to detect the effect of intrinsic factors contributing to gene expression

355 heterogeneity such as axolotl regenerative development inherent asynchrony. Most importantly,

356 despite the potential contribution of regenerative development asynchrony to gene expression

357 heterogeneity, post-amputational cvGEBIs are significantly negative 1.5 days post-amputation

358 onwards, as it would be expected if samples encompassing these timepoints were populated by

359 dedifferentiated cells with extensively relaxed chromatin.

360

361 Whether the significant reduction in gene expression heterogeneity here reported truly reflected

362 the presence of a significant number of dedifferentiated cells with extensively relaxed chromatin

363 within post-amputational forearm blastemas would require independent corroboration using 
364 methodologies that go beyond the scope of this article. However, further analyses of the dataset

365 under study resulted in other observations that favor this hypothesis over other obvious

366 explanations for the observed decline in gene expression heterogeneity.

367

368 Gene expression heterogeneity dynamics along axolotl forearm regenerative development

369 cannot be explained by a generalized increase in gene expression. In principle, the decrease in

370 gene expression heterogeneity here detected for axolotl forelimb regenerative development could

371 be explained without assuming that intervening cells undergo extensive chromatin relaxation. It

372 has been shown that cells participating in regenerative processes tend to show a generalized

373 increase in gene expression (Percharde et al. 2017). Since, gene expression heterogeneity is

374 known to negatively correlate with gene expression level (Liu et al. 2016; Symmons \& Raj

375 2016), it would be expected that blastemal samples showed a reduction in gene expression

376 heterogeneity regardless of intervening cells undergoing extensive chromatin relaxation.

377

378 To directly test whether the reduction in gene expression heterogeneity here detected resulted

379 from blastemal cells generalized increase in gene expression, first, I proceed to study the

380 dynamics of the level of gene expression for all genes in the dataset under study. Unrestricted

381 MCW tests using transcript abundance mean per probeset and timepoint as proxy for their level

382 of gene expression were used to quantify gene expression level biases across the transcriptome

383 for TX vs T0 comparisons. GEBIs calculated using transcript abundance means are referred to as

384 mGEBIs. Consistent with the possibility that blastemal cell showed a generalized increase in

385 gene expression, mGEBIs become positive and significantly different from mGEBIs expected by

386 chance 2 days after amputation (Figure 1, and Table S2). In other words, genes tend to become 
387 significantly overexpressed post-amputationally starting 2 days after amputation. Furthermore, at

388 a first glance, cvGEBI and mGEBI temporal dynamics are quasi-specular, underscoring the

389 possibility that gene expression heterogeneity decrease along axolotl forearm regenerative

390 development were just a consequence of blastemal cell generalized increase in gene expression.

391 However, mGEBIs become significantly positive 2 days after amputation, with a small delay

392 with regard to cvGEBIs becoming significantly negative 1.5 days after amputation. Such delay is

393 more consistent with the possibility that blastemal cell generalized increase in gene expression

394 was a consequence of intervening cells extensive chromatin relaxation that might contribute to

395 the earlier significant decrease in gene expression heterogeneity, than the latter being a side

396 consequence of blastemal cell generalized increase in gene expression.

397

398 To further study the interrelationship of the dynamics for gene expression level and

399 heterogeneity along axolotl forearm regenerative development, three different variations of

$400 \mathrm{MCW}$ tests for transcript abundance $\mathrm{CV}$ and $\mathrm{TX} v s \mathrm{~T} 0$ pairwise comparisons were used (Figure

401 S2). Unrestricted MCW tests were used to ascertain if observed biases for transcript abundance

402 CV were significantly different from those biases expected just by chance. Timepoint-restricted

$403 \mathrm{MCW}$ tests were used to ascertain if observed biases for transcript abundance CV were

404 significantly different from those expected if gene expression heterogeneity was exclusively

405 dependent on factors acting on the transcriptome as a whole. Finally, expression-restricted MCW

406 tests were used to ascertain if observed biases for transcript abundance CV were significantly

407 different from those expected if gene expression heterogeneity was exclusively dependent on the

408 variation in the mean level of expression for each probeset. If post-amputational gene expression

409 heterogeneity dynamics was just a mere reflection of blastemal cell generalized increase in gene 
410 expression it would be expected that observed cvGEBIs were not significantly distinguishable

411 from simulated GEBIs for expression-restricted MCW tests. On the contrary, if post-

412 amputational gene expression heterogeneity dynamics was dependent on factors acting on the

413 whole transcriptome, it would be expected that observed cvGEBIs were not significantly

414 distinguishable from simulated GEBIs for timepoint-restricted MCW tests.

415

416 Observed cvGEBIs were significantly different from simulated ones for all timepoints using any

417 of the three MCW test designs (Figure 2A, and Table S2), suggesting that chance, factors acting

418 on the transcriptome as a whole, or the variation in the level of expression cannot explain on

419 their own gene expression heterogeneity dynamics along axolotl forearm regenerative

420 development. The proportional cumulative area under the curve $\left(\mathrm{PC}_{\mathrm{AUC}}\right)$ using simulated

421 cvGEBIs with the closest value to observed cvGEBIs for each timepoint and MCW test design

422 was calculated to estimate how much of gene expression heterogeneity dynamics could be

423 explained by chance, factors acting on the transcriptome as a whole, or the variation in the level

424 of expression (Figure 2B, and Table S4). The fraction of observed cvGEBIs dynamics that could

425 be explained by the variation in the mean level of expression of each probeset under study is

426 barely higher than what it could be explained just by chance alone (18\% versus $16 \%)$. In stark

427 contrast, factors acting on the transcriptome as a whole could explain up to $60 \%$ of observed

428 cvGEBIs temporal dynamics. Considering the multidimensionality of the dataset, the potential

429 interdependencies that might exist between its elements, i.e., genes that regulate other genes, or

430 the effect of other factors that contributed to gene expression heterogeneity like developmental

431 asynchrony, the fact that simulated cvGEBIs obtained using timepoint-restricted MCWs could

432 explain $60 \%$ of observed gene expression heterogeneity dynamics is remarkable. These results 
433 strongly suggest that gene expression heterogeneity dynamics along axolotl forearm regenerative

434 development cannot be easily attributed to blastemal cell generalized increase in gene

435 expression. Instead, these results are more consistent with the possibility that axolotl forearm

436 regenerative development concurred with an extensive decrease in gene expression heterogeneity

437 associated to intervening cells undergoing extensive chromatin relaxation, which could be

438 conducive for the expression increase of many genes throughout the genome.

440 Gene expression heterogeneity decrease for axolotl forearm regenerative development is

441 consistent with blastemal cell dedifferentiation. The decrease in gene expression heterogeneity

442 for axolotl forearm regenerative development here detected could be explained even if no

443 intervening cell dedifferentiated. Whichever is the origin of blastemal cells, they are expected to

444 encompass a more homogenous pool of cell types than those represented in samples taken at the

445 moment of the amputation, and, immediately after that, when a diverse number of processes are

446 elicited to heal the wound (McCusker et al. 2015a). If the decline in gene expression

447 heterogeneity here documented was caused by the relative enrichment of cells from a reduced

448 number of cell types, it would be expected that genes supporting the earliest decline in gene

449 expression heterogeneity resembled the transcriptional profile typical of the few cell types there

450 represented.

451

452 To ascertain whether the extensive decrease in gene expression heterogeneity here detected was

453 dependent on the relative enrichment in one or few cell types, I proceed to functionally

454 characterized those probesets that better resemble the sharp decrease in gene expression

455 heterogeneity between 1 and 1.5 days post-amputation (Figure 1, and Table S2). Probesets in the 
456 dataset were ranked with regard to transcript abundance CV fold change for 1 and 1.5 days post-

457 amputation timepoints. The genes corresponding to this prioritized list of probesets and 10 more

458 lists of genes generated by randomly permutating probesets in the dataset with no other

459 restriction were used to identify significant Gene Ontology (GO) enrichments using GOrilla and

460 REVIGO (Eden et al. 2009; Supek et al. 2011). Only GO terms "mitotic cell cycle process"

461 (GO:1903047) and "chromatin organization" (GO:0006325) appeared significantly enriched for

462 probesets with a decrease in gene expression heterogeneity between 1 and 1.5 days post-

463 amputation (Tables 1, and S5). Also, just 10 genes were found supporting the enrichment of both

464 terms out of the 155 genes that support either enrichment, suggesting that the sets of genes

465 supporting each GO enrichment are largely different.

466

467 To further characterize the temporal dynamics of transcripts associated to "mitotic cell cycle

468 process" and "chromatin organization" GO terms with regard to the whole transcriptome, I used

469 a new variation of the MCW test. Functionally-restricted MCW tests proceed by calculating

470 cvGEBI and mGEBI for probesets associated to genes classified in "mitotic cell cycle process"

471 and "chromatin organization" GO terms before and after randomly rearranging functional subset

472 tags for the whole dataset, respectively (Figure S2). For both GO terms, the expected sharp

473 decrease in transcript abundance $\mathrm{CV}$ between 1 and 1.5 days post-amputation is followed by a

474 sharp increase in transcript abundance mean between 2 and 3 days post-amputation (Figure 3,

475 and Table S2). Consistent with these trends, the original characterization of Voss dataset showed

476 significant enrichments for GO terms related with cell cycle and chromosome organization for

477 probesets with a statistically significant difference in transcript abundance between 2 and 3 days

478 post-amputation timepoints (Voss et al. 2015a). 
480 "Mitotic cell cycle process" and "chromatin organization" GO term enrichment for probesets

481 better supporting the sharp decrease in gene expression heterogeneity between 1 and 1.5 days

482 post-amputation timepoints, and the transcriptomic dynamics of probesets for genes associated to

483 these two terms along axolotl forearm regenerative development does not seem to be consistent

484 with the possibility that early stages of regenerative development coursed with a relative

485 enrichment in one or few cell types. Instead, these trends seem to relate better with the possibility

486 that intervening cells undergo the earliest steps of transformations expected for dedifferentiating

487 cells, namely re-entry in cell cycle and chromatin reorganization. If a reduction in gene

488 expression heterogeneity followed by an increase in the expression of those genes that might

489 drive the process of cell dedifferentiation reflected that chromosome regions encompassing these

490 genes were the first to undergo chromatin relaxation for cells leading regenerative responses

491 suggests that studies focused on the dynamics of the chromatin context for those genes that show

492 the earliest decline in gene expression heterogeneity along regenerative development process

493 could be of great help to better understanding how regenerative development is elicited in

494 response to injury.

495

496 DISCUSSION

497 In the present article, using already available transcriptomic data for axolotl regenerative

498 development and the assumption that such regenerative processes concurs with cell

499 dedifferentiation, very preliminary support is given to the proposal that cell dedifferentiation

500 concurs with an extensive decrease in gene expression heterogeneity (Díaz-Castillo 2017b; Voss

501 et al. 2015a). Although here I used a dataset that has already been characterized, the analyses 
502 here presented differ from the original characterization of the dataset mostly because they focus

503 on the study of changes in gene expression heterogeneity; changes to the mean level of

504 expression are used only accessorily. Another important difference of the present study from the

505 original characterization and other transcriptomic studies consist in the use of MCW tests, which

506 permits characterizing extensive transcriptomic trends taking into consideration all the genes

507 encompassed by large datasets, instead of just those showing differences beyond thresholds

508 chosen arbitrarily.

509

510 An important caveat to the analyses here presented relates with the increasing notion that

511 commonly used analytical methodologies to study transcriptome dynamics might not fairly

512 represent cases in which the transcriptional output of all or most genes in the genome is

513 concertedly increased (Percharde et al. 2017). The use of normalization methods based on the

514 assumption that the expression of "housekeeping" genes or the general transcription output of the

515 nucleus is largely invariable between the assayed conditions, and the difficulty in precisely

516 controlling the number of cells contained in samples for complex and very dynamic processes

517 such as regenerative development represent the main difficulties to ensure a fair consideration of

518 cell transcriptional output for transcriptomic analyses (Percharde et al. 2017). Future analyses,

519 probably focused on single cells, will be needed to assess the impact of this methodological

520 caveat for the study of transcriptome dynamics in general, and for these circumstances that

521 concur with a generalized increase in gene expression in particular.

522

523 How many and which, if any, cells dedifferentiate upon injury along regenerative development

524 processes, and if such dedifferentiation indeed concur with extensive chromatin relaxation 
525 driving to an extensive decrease in gene expression heterogeneity require independent

526 confirmation. The possibility that changes in gene expression heterogeneity could approximate

527 changes in chromatin configuration for dedifferentiated cells might be of wide interest for

528 biomedical and biotechnological areas based on cell dedifferentiation such as regenerative

529 development, cancer, human iPSCs, or plant somatic embryogenesis. Here, I proceed to briefly

530 discuss how the study of gene expression heterogeneity dynamics might help developing

531 methodologies to monitor and/or control cell dedifferentiation-based processes, and how a

532 transitional reduction in gene expression heterogeneity for dedifferentiating cells can promote

533 undesired longer-term phenotypic heterogeneity.

534

535 The study of gene expression heterogeneity can be useful for the characterization of processes that concur with cell dedifferentiation, and to engineer approaches for their

monitoring and modulation. Whole transcriptome analysis is now a common tool to identify relevant changes in transcript abundance reflecting natural or experimental cues. Often, these analyses are based on the dubious assumption that significant changes in transcript abundance

540 directly reflect equally significant changes in the function/effect of the very transcript or the

541 protein it codes for. However, the study of transcript abundance dynamics permits directly

542 addressing only a small fraction of the regulatory mechanisms needed for the generation of

543 active genetic products. Transcript spatial distribution, and translation, or protein post-

544 translational modification, folding, spatial distribution, and coordinated functionality with other

545 genetic products remain largely unapproachable using only transcriptomic analyses. Since all

546 these steps in the de novo synthesis of active genetic products are susceptible to be regulated

547 independently from regulatory mechanisms modulating transcript abundance, it is possible that 
548 very obvious changes in transcript abundance are phenotypically insignificant because they are

549 buffered by the system, or genes for which no significant change is detected at a transcriptional

550 level had key roles in certain processes because of regulatory inputs acting at the protein level.

551 This limitation is particularly troublesome when transcriptomic analyses are used to distinguish

552 genes that drive a particular process from those that reflect the progression of the process in

553 question, i.e., drivers versus effectors. While such distinction might be meaningless for the

554 identification of diagnostic markers for biological processes, it is key for the basic

555 characterization of such processes and the efficient engineering of strategies that afford in vivo

556 monitoring or modulating their progress.

557

558 Gene expression heterogeneity is not directly informative about the abundance of active genetic

559 products, and, therefore, its use to infer functional changes in the process under consideration is

560 also very limited. However, gene expression heterogeneity dynamics might be highly

561 informative about regulatory mechanisms acting on chromatin accessibility, transcription, and

562 transcript degradation. In particular, the integration of gene expression heterogeneity dynamics

563 with proxies for higher order chromatin organization could be of great value to identify

564 chromosome regions encompassing genes that undergo the earliest changes along naturally-

565 occurring or induced cell dedifferentiation, and the characterization of the regulatory elements

566 mediating such changes. Furthermore, the possibility of identifying chromosome regions

567 showing the earliest changes associated to cell dedifferentiation would afford using them as

568 target for the insertion of engineered genetic constructs that help better monitoring or even

569 modulating processes that concur with cell dedifferentiation. For example, in the context of

570 regenerative development in response to injury, chromosome regions undergoing the earliest 
571 chromatin relaxation along cell dedifferentiation could be targeted for the insertion of genetic

572 constructs coding for visible markers that helped identifying which cells within a complex tissue

573 are susceptible to dedifferentiate in response to injury, or monitoring the progress of regenerative

574 responses in non-invasive ways. Alternatively, the same regions could be targeted for the

575 insertion of constructs coding for RNAs of proteins that by stimulating or interfering with key

576 elements of cell dedifferentiation-based processes permitted modulating the course of such

577 processes and improve their efficiency or minimize detrimental side effects.

578

579 A transitional reduction in gene expression heterogeneity for dedifferentiating cells could

580 promote a long-term phenotypic heterogeneity. A troublesome aspect of processes that concur

581 with cell dedifferentiation is the accentuated heterogeneity detected at genetic, epigenetic, and

582 phenotypic levels (Almendro et al. 2013; Burrell et al. 2013; Fossati et al. 2016; Krishna et al.

583 2016; Ling et al. 2015; Meacham \& Morrison 2013). Such heterogeneities are of great concern

584 because they can limit the response to treatment of cancerous cell, or the reproducibility of

585 healthy clonal tissues and individuals derived from animal and plant induced dedifferentiated

586 cells (Almendro et al. 2013; Burrell et al. 2013; Fossati et al. 2016; Krishna et al. 2016; Ling et

587 al. 2015; Meacham \& Morrison 2013). Intriguingly, a chromatin relaxation-based extensive

588 reduction in gene expression heterogeneity for dedifferentiated cells could promote long-term

589 phenotypic heterogeneities following cell dedifferentiation by increasing genetic and epigenetic

590 mutagenic potential and the phenotypic relevance of preexisting or newly generated mutations

591 for dedifferentiated cells.

592

593 On one hand, an extensive chromatin relaxation-based surge of DNA accessibility might result in 
594 a larger number of coding or regulatory loci being susceptible to insults that result in genetic

595 mutations, but also the activation of genetic elements that can directly intervene in mutagenic

596 events such as those transposable elements populating regions of highly compacted chromatin.

597 Indeed, abundant reports exist for accentuated genetic variation, and activities of transposable

598 elements in processes concurring with cell dedifferentiation (Cooper et al. 2017; Jiang et al.

599 2011; Ling et al. 2015; Macia et al. 2015; Wang \& Wang 2012; Zhu et al. 2012a). Furthermore,

600 the increased activity of repair machineries that help protecting the integrity of the genome in

601 dedifferentiated cells might evidence their enhanced potential for genetic mutagenesis (Cooper et

602 al. 2017). Also, 5-methylcytosine (5mC) is highly prone to spontaneously deaminate into

603 thymine (T), which if uncorrected would cause both genetic and epigenetic mutations (Bellacosa

604 \& Drohat 2015; Cortazar et al. 2007). The restoration of 5-methylcytosine is not straightforward

605 and requires a concatenation of enzymes that recognize thymine:guanidine (T:G) mismatches,

606 excise T, restore the original cytosine (C), and methylate the restored C (Bellacosa \& Drohat

607 2015; Cortazar et al. 2007). The key enzyme for 5mC deamination repair is thymine glycosylase

608 (TDG), which is known to be considerably inefficient (Bellacosa \& Drohat 2015; Cortazar et al.

609 2007). The accentuated tendency to deaminate of $5 \mathrm{mC}$, the complicated way of restoring $5 \mathrm{mC}$,

610 and the inefficiency of some of the elements required for $5 \mathrm{mC}$ restoration suggest that for any

611 given methylated locus in a population of isogenic cells in the same environment there would be

612 a variable assortment of cells showing $5 \mathrm{mC}$, $\mathrm{T}$, or C. Since the limiting step for $5 \mathrm{mC}$

613 deamination is DNA melting (Fryxell \& Moon 2005), processes more permissive for DNA

614 melting, such as dedifferentiated cells extensive chromatin relaxation, could result in an

615 accentuated and ultimately stochastic variation in DNA methylation and 5mC deamination-based

616 mutations. 
618 On the other hand, beyond the direct effect chromatin relaxation in dedifferentiated cells might

619 have on the genetic and epigenetic mutagenic potential, a transitional chromatin relaxation-

620 dependent extensive decrease in gene expression heterogeneity can also promote a long-term

621 phenotypic heterogeneity by enhancing the phenotypic relevance of pre-existing and newly

622 generated genetic and epigenetic mutations. Genetic capacitance refers to the accumulation and

623 release of genetic variation in a cryptic state, i.e., not causing phenotypic variation (Gibson \&

624 Reed 2008; Masel \& Trotter 2010; Paaby \& Rockman 2014). In recent years, it has been

625 suggested that chromatin compaction-dependent gene expression heterogeneity promotes genetic

626 capacitance because it results in a spectrum of stochastic phenotypes from which some genetic-

627 based phenotypes might be indistinguishable (Díaz-Castillo 2015; Díaz-Castillo 2017a). Such

628 cryptic genetic variation would be undetectable for selective forces, and, therefore allowed to

629 fluctuate randomly within biological populations. Generalized differences in chromatin

630 compaction modulating gene expression heterogeneity, and, with it, genetic capacitance, have

631 been proposed to importantly contribute to sexually dimorphic traits in metazoans and

632 differences in the spatiotemporal dynamics of natural populations from species with different

633 amounts of junk DNA (Díaz-Castillo 2015; Díaz-Castillo 2017a).

634

635 If cell dedifferentiation truly concurred with an extensive decrease in chromatin compaction-

636 dependent gene expression heterogeneity, it could be expected that such decrease resulted in a

637 reduction in genetic capacitance (Figure 4). In other words, due to the extensive decrease in gene

638 expression heterogeneity, the spectrum of stochastic phenotypes would become narrower, and,

639 consequently, preexisting cryptic or new genetic and epigenetic mutations would become 
640 phenotypically relevant (Figure 3). Revealed genetic and epigenetic mutations would contribute

641 to a phenotypic plasticity in dedifferentiated cells noticeable in the absence of any other cue, or

642 upon signaling from their environments or the action of external treatments. The growing

643 literature on the relevance of stochastic events commonly occurring in early development could

644 support the possibility that un/dedifferentiated cells showed limited genetic capacitance because,

645 at least in part, their extensive chromatin relaxation, and, therefore, being more susceptible to

646 manifest plasticity inherently and/or in response to external cues (Losick \& Desplan 2008; Vogt

647 2015).

648

649 In summary, an extensive reduction in gene expression heterogeneity for dedifferentiated cells is

650 not only not conflicting with the multiple reports on accentuated genetic, epigenetic, and

651 phenotypic heterogeneities for natural or induced processes that concur with cell

652 dedifferentiation, but it could be argued that the extensive chromatin relaxation typical of

653 dedifferentiated cells might be the ultimate cause for these apparently opposing heterogeneity 654 trends.

655

656 CONCLUDING REMARKS

657 The high precision and resolution achieved with modern techniques is providing us with very

658 large collections of data for all sorts of biological systems and processes. Less progress has been

659 made analytically to squeeze as much biologically relevant information as possible from such

660 highly multidimensional datasets. Although the study of biological variation observed in the

661 absence of genetic and environmental cues and its usefulness for basic and applied biology is

662 still very much underrated, an appreciation for the study of mechanisms that cause biological 
663 variation and its potential for biomedical applications is starting to grow (Rosenberg \& Queitsch

664 2014). That cell dedifferentiation might concur with an extensive reduction in gene expression

665 heterogeneity might be of great interest for biomedical and biotechnological processes that

666 depend on cell dedifferentiation, i.e., regenerative development, cancer, human iPSCs, or plant

667 somatic embryogenesis. The study of cell dedifferentiation-based gene expression heterogeneity

668 dynamics opens a complementary way to characterize these processes, especially in their earliest

669 stages, and lay the foundation to newer more precise tools that help monitoring and modulating

670 them. Furthermore, the possibility that a reduction in gene expression heterogeneity can promote

671 long-term phenotypic heterogeneity following cell dedifferentiation points to the possibility of

672 identifying key elements to target in an attempt to minimize the adverse effect such phenotypic

673 heterogeneity might have for the response to treatment, or the reproducibility of healthy clonal

674 tissues and individuals. More generally, the utilization of appropriate analytical methods to

675 characterize gene expression heterogeneity dynamics as an adjunct to already existing methods

676 might help accessing layers of information buried in today's multidimensional datasets and offer

677 a more representative understanding of biological systems and processes.

678

679 ACKNOWLEDGMENTS

680 I want to thank Min Zhao, and three anonymous reviewers for their constructive criticism during 681 the revision of this article. I want to thank Randal Voss and Zeba Wunderlich for valuable 682 comments during the preparation of this article. I want to express my deepest gratitude to Sue 683 Bryant, David Gardiner, and Kate McCusker for valuable comments during the preparation of 684 this article, and their generosity and support during my acclimation to the study of axolotl 
685 regenerative development. I want to express my eternal gratitude to Raquel Chamorro-Garcia for

686

687

688

689

690

691

692

693

694

695

696

697

698

699

700

701

702

703

704

705

706

707

708

709

710

711

712

713

714

715

716

717

718

719

720

721

722

723

724

725

726

valuable comments during the preparation of this article, and her unfailing support.

\section{REFERENCES}

Ackermann M. 2015. A functional perspective on phenotypic heterogeneity in microorganisms. Nat Rev Microbiol 13:497-508. 10.1038/nrmicro3491

Almendro V, Marusyk A, and Polyak K. 2013. Cellular heterogeneity and molecular evolution in cancer. Annu Rev Pathol 8:277-302. 10.1146/annurev-pathol-020712-163923

Altschuler SJ, and Wu LF. 2010. Cellular heterogeneity: do differences make a difference? Cell 141:559-563. 10.1016/j.cell.2010.04.033

Barrett T, Wilhite SE, Ledoux P, Evangelista C, Kim IF, Tomashevsky M, Marshall KA, Phillippy KH, Sherman PM, Holko M, Yefanov A, Lee H, Zhang N, Robertson CL, Serova N, Davis S, and Soboleva A. 2013. NCBI GEO: archive for functional genomics data sets--update. Nucleic Acids Res 41:D991-995. 10.1093/nar/gks1193

Bellacosa A, and Drohat AC. 2015. Role of base excision repair in maintaining the genetic and epigenetic integrity of CpG sites. DNA Repair (Amst) 32:33-42.

10.1016/j.dnarep.2015.04.011

Bryant DM, Johnson K, DiTommaso T, Tickle T, Couger MB, Payzin-Dogru D, Lee TJ, Leigh ND, Kuo TH, Davis FG, Bateman J, Bryant S, Guzikowski AR, Tsai SL, Coyne S, Ye WW, Freeman RM, Jr., Peshkin L, Tabin CJ, Regev A, Haas BJ, and Whited JL. 2017. A Tissue-Mapped Axolotl De Novo Transcriptome Enables Identification of Limb Regeneration Factors. Cell Rep 18:762-776. 10.1016/j.celrep.2016.12.063

Burrell RA, McGranahan N, Bartek J, and Swanton C. 2013. The causes and consequences of genetic heterogeneity in cancer evolution. Nature 501:338-345. 10.1038/nature12625

Cooper DJ, Chen IC, Hernandez C, Wang Y, Walter CA, and McCarrey JR. 2017. Pluripotent cells display enhanced resistance to mutagenesis. Stem Cell Res 19:113-117. 10.1016/j.scr.2016.12.029

Cortazar D, Kunz C, Saito Y, Steinacher R, and Schar P. 2007. The enigmatic thymine DNA glycosylase. DNA Repair (Amst) 6:489-504. 10.1016/j.dnarep.2006.10.013

Díaz-Castillo C. 2015. Evidence for a sexual dimorphism in gene expression noise in metazoan species. PeerJ 3:e750. 10.7717/peerj.750

Díaz-Castillo C. 2017a. Junk DNA Contribution to Evolutionary Capacitance Can Drive Species Dynamics. Evolutionary Biology 44:190-205. 10.1007/s11692-016-9404-5

Díaz-Castillo C. 2017b. Theorizing about gene expression heterogeneity patterns after cell dedifferentiation and their potential value for regenerative engineering. In: Gardiner DM, ed. Regenerative Engineering and Developmental Biology: Principles and Applications. Florida: CRC Press, 351-360.

Eden E, Navon R, Steinfeld I, Lipson D, and Yakhini Z. 2009. GOrilla: a tool for discovery and visualization of enriched GO terms in ranked gene lists. BMC Bioinformatics 10:48. 10.1186/1471-2105-10-48

El-Badawy A, and El-Badri N. 2015. Regulators of pluripotency and their implications in regenerative medicine. Stem Cells Cloning 8:67-80. 10.2147/SCCAA.S80157 
727

728

729

730

731

732

733

734

735

736

737

738

739

740

741

742

743

744

745

746

747

748

749

750

751

752

753

754

755

756

757

758

759

760

761

762

763

764

765

766

767

768

769

770

771
Elgin SC, and Reuter G. 2013. Position-effect variegation, heterochromatin formation, and gene silencing in Drosophila. Cold Spring Harb Perspect Biol 5:a017780. 10.1101/cshperspect.a017780

Eo SH, Doyle JM, Hale MC, Marra NJ, Ruhl JD, and DeWoody JA. 2012. Comparative transcriptomics and gene expression in larval tiger salamander (Ambystoma tigrinum) gill and lung tissues as revealed by pyrosequencing. Gene 492:329-338. 10.1016/j.gene.2011.11.018

Feher A. 2015. Somatic embryogenesis - Stress-induced remodeling of plant cell fate. Biochim Biophys Acta 1849:385-402. 10.1016/j.bbagrm.2014.07.005

Fossati V, Jain T, and Sevilla A. 2016. The silver lining of induced pluripotent stem cell variation. Stem Cell Investig 3:86. 10.21037/sci.2016.11.16

Fryxell KJ, and Moon WJ. 2005. CpG mutation rates in the human genome are highly dependent on local GC content. Mol Biol Evol 22:650-658. 10.1093/molbev/msi043

Gearhart MD, Erickson JR, Walsh A, and Echeverri K. 2015. Identification of Conserved and Novel MicroRNAs during Tail Regeneration in the Mexican Axolotl. Int J Mol Sci 16:22046-22061. 10.3390/ijms160922046

Gibson G, and Reed LK. 2008. Cryptic genetic variation. Curr Biol 18:R989-990. 10.1016/j.cub.2008.08.011

Grafi G, and Barak S. 2015. Stress induces cell dedifferentiation in plants. Biochim Biophys Acta 1849:378-384. 10.1016/j.bbagrm.2014.07.015

Jiang C, Mithani A, Gan X, Belfield EJ, Klingler JP, Zhu JK, Ragoussis J, Mott R, and Harberd NP. 2011. Regenerant Arabidopsis lineages display a distinct genome-wide spectrum of mutations conferring variant phenotypes. Curr Biol 21:1385-1390.

10.1016/j.cub.2011.07.002

Jiang F, Zhu J, and Liu HL. 2013. Protoplasts: a useful research system for plant cell biology, especially dedifferentiation. Protoplasma 250:1231-1238. 10.1007/s00709-013-0513-z

King BL, and Yin VP. 2016. A Conserved MicroRNA Regulatory Circuit Is Differentially Controlled during Limb/Appendage Regeneration. PLoS One 11:e0157106. 10.1371/journal.pone.0157106

Knapp D, Schulz H, Rascon CA, Volkmer M, Scholz J, Nacu E, Le M, Novozhilov S, Tazaki A, Protze S, Jacob T, Hubner N, Habermann B, and Tanaka EM. 2013. Comparative transcriptional profiling of the axolotl limb identifies a tripartite regeneration-specific gene program. PLoS One 8:e61352. 10.1371/journal.pone.0061352

Komin N, and Skupin A. 2017. How to address cellular heterogeneity by distribution biology. Current Opinion in Systems Biology 3:154-160.

https://doi.org/10.1016/j.coisb.2017.05.010

Kragl M, Knapp D, Nacu E, Khattak S, Maden M, Epperlein HH, and Tanaka EM. 2009. Cells keep a memory of their tissue origin during axolotl limb regeneration. Nature 460:60-65. 10.1038 /nature 08152

Krause MN, Sancho-Martinez I, and Izpisua Belmonte JC. 2015. Understanding the molecular mechanisms of reprogramming. Biochem Biophys Res Commun. 10.1016/j.bbrc.2015.11.120

Krishna H, Alizadeh M, Singh D, Singh U, Chauhan N, Eftekhari M, and Sadh RK. 2016. Somaclonal variations and their applications in horticultural crops improvement. 3 Biotech 6:54. 10.1007/s13205-016-0389-7 
772

773

774

775

776

777

778

779

780

781

782

783

784

785

786

787

788

789

790

791

792

793

794

795

796

797

798

799

800

801

802

803

804

805

806

807

808

809

810

811

812

813

814

815

816
Lee K, Park OS, Jung SJ, and Seo PJ. 2015. Histone deacetylation-mediated cellular dedifferentiation in Arabidopsis. J Plant Physiol 191:95-100. 10.1016/j.jplph.2015.12.006

Li M, and Belmonte JC. 2017. Ground rules of the pluripotency gene regulatory network. Nat Rev Genet 18:180-191. 10.1038/nrg.2016.156

Ling S, Hu Z, Yang Z, Yang F, Li Y, Lin P, Chen K, Dong L, Cao L, Tao Y, Hao L, Chen Q, Gong Q, Wu D, Li W, Zhao W, Tian X, Hao C, Hungate EA, Catenacci DV, Hudson RR, Li WH, Lu X, and Wu CI. 2015. Extremely high genetic diversity in a single tumor points to prevalence of non-Darwinian cell evolution. Proc Natl Acad Sci US A 112:E6496-6505. 10.1073/pnas.1519556112

Liu J, Francois JM, and Capp JP. 2016. Use of noise in gene expression as an experimental parameter to test phenotypic effects. Yeast 33:209-216. 10.1002/yea.3152

Losick R, and Desplan C. 2008. Stochasticity and cell fate. Science 320:65-68. $10.1126 /$ science. 1147888

Macia A, Blanco-Jimenez E, and Garcia-Perez JL. 2015. Retrotransposons in pluripotent cells: Impact and new roles in cellular plasticity. Biochim Biophys Acta 1849:417-426. 10.1016/j.bbagrm.2014.07.007

Maggert KA, and Golic KG. 2002. The Y chromosome of Drosophila melanogaster exhibits chromosome-wide imprinting. Genetics 162:1245-1258.

Martinez O, and Reyes-Valdes MH. 2008. Defining diversity, specialization, and gene specificity in transcriptomes through information theory. Proc Natl Acad Sci U S A 105:9709-9714. 10.1073/pnas.0803479105

Martinez O, Reyes-Valdes MH, and Herrera-Estrella L. 2010. Cancer reduces transcriptome specialization. PLoS One 5:e10398. 10.1371/journal.pone.0010398

Masel J, and Trotter MV. 2010. Robustness and evolvability. Trends Genet 26:406-414. 10.1016/j.tig.2010.06.002

McCusker C, Bryant SV, and Gardiner DM. 2015a. The axolotl limb blastema: cellular and molecular mechanisms driving blastema formation and limb regeneration in tetrapods. Regeneration (Oxf) 2:54-71. 10.1002/reg2.32

McCusker CD, Athippozhy A, Díaz-Castillo C, Fowlkes C, Gardiner DM, and Voss SR. 2015 b. Positional plasticity in regenerating Amybstoma mexicanum limbs is associated with cell proliferation and pathways of cellular differentiation. BMC Dev Biol 15:45. 10.1186/s 12861-015-0095-4

Meacham CE, and Morrison SJ. 2013. Tumour heterogeneity and cancer cell plasticity. Nature 501:328-337. 10.1038/nature12624

Merrell AJ, and Stanger BZ. 2016. Adult cell plasticity in vivo: de-differentiation and transdifferentiation are back in style. Nat Rev Mol Cell Biol 17:413-425. 10.1038/nrm.2016.24

Monaghan JR, Athippozhy A, Seifert AW, Putta S, Stromberg AJ, Maden M, Gardiner DM, and Voss SR. 2012. Gene expression patterns specific to the regenerating limb of the Mexican axolotl. Biol Open 1:937-948. 10.1242/bio.20121594

Monaghan JR, Epp LG, Putta S, Page RB, Walker JA, Beachy CK, Zhu W, Pao GM, Verma IM, Hunter T, Bryant SV, Gardiner DM, Harkins TT, and Voss S. 2009. Microarray and cDNA sequence analysis of transcription during nerve-dependent limb regeneration. BMC biology 7:1. DOI: 10.1186/1741-7007-7-1 
817 Paaby AB, and Rockman MV. 2014. Cryptic genetic variation: evolution's hidden substrate. Nat $818 \quad$ Rev Genet 15:247-258. 10.1038/nrg3688

819 Pai VP, Martyniuk CJ, Echeverri K, Sundelacruz S, Kaplan DL, and Levin M. 2016. Genome-

820

821

822

823

824

825

826

827

828

829

830

831

832

833

834

835

836

837

838

839

840

841

842

843

844

845

846

847

848

849

850

851

852

853

854

855

856

857

858

859

860

861 wide analysis reveals conserved transcriptional responses downstream of resting potential change in Xenopus embryos, axolotl regeneration, and human mesenchymal cell differentiation. Regeneration (Oxf) 3:3-25. 10.1002/reg2.48

Percharde M, Bulut-Karslioglu A, and Ramalho-Santos M. 2017. Hypertranscription in Development, Stem Cells, and Regeneration. Dev Cell 40:9-21. 10.1016/j.devcel.2016.11.010

Ponomareva LV, Athippozhy A, Thorson JS, and Voss SR. 2015. Using Ambystoma mexicanum (Mexican axolotl) embryos, chemical genetics, and microarray analysis to identify signaling pathways associated with tissue regeneration. Comp Biochem Physiol C Toxicol Pharmacol 178:128-135. 10.1016/j.cbpc.2015.06.004

Rivas G, and Minton AP. 2016. Macromolecular Crowding In Vitro, In Vivo, and In Between. Trends Biochem Sci 41:970-981. 10.1016/j.tibs.2016.08.013

Rosenberg SM, and Queitsch C. 2014. Medicine. Combating evolution to fight disease. Science 343:1088-1089. 10.1126/science. 1247472

Sabin K, Santos-Ferreira T, Essig J, Rudasill S, and Echeverri K. 2015. Dynamic membrane depolarization is an early regulator of ependymoglial cell response to spinal cord injury in axolotl. Dev Biol 408:14-25. 10.1016/j.ydbio.2015.10.012

Sandoval-Guzman T, Wang H, Khattak S, Schuez M, Roensch K, Nacu E, Tazaki A, Joven A, Tanaka EM, and Simon A. 2014. Fundamental differences in dedifferentiation and stem cell recruitment during skeletal muscle regeneration in two salamander species. Cell Stem Cell 14:174-187. 10.1016/j.stem.2013.11.007

Seifert AW, Monaghan JR, Voss SR, and Maden M. 2012. Skin regeneration in adult axolotls: a blueprint for scar-free healing in vertebrates. PLoS One 7:e32875. 10.1371/journal.pone.0032875

Sosnik J, Vieira WA, Webster KA, Siegfried KR, and McCusker CD. 2017. A new and improved algorithm for the quantification of chromatin condensation from microscopic data shows decreased chromatin condensation in regenerating axolotl limb cells. PLoS One 12:e0185292. 10.1371/journal.pone.0185292

Sousounis K, Athippozhy AT, Voss SR, and Tsonis PA. 2014. Plasticity for axolotl lens regeneration is associated with age-related changes in gene expression. Regeneration (Oxf) 1:47-57. 10.1002/reg2.25

Stewart R, Rascón CA, Tian S, Nie J, Barry C, Chu L-F, Ardalani H, Wagner RJ, Probasco MD, Bolin JM, Leng N, Sengupta S, Volkmer M, Habermann B, Tanaka EM, Thomson JA, and Dewey CN. 2013. Comparative RNA-seq Analysis in the Unsequenced Axolotl: The Oncogene Burst Highlights Early Gene Expression in the Blastema. PLoS Computational Biology 9:e1002936. DOI: 10.1371/journal.pcbi.1002936

Sugiyama M. 2015. Historical review of research on plant cell dedifferentiation. J Plant Res 128:349-359. 10.1007/s10265-015-0706-y

Supek F, Bosnjak M, Skunca N, and Smuc T. 2011. REVIGO summarizes and visualizes long lists of gene ontology terms. PLoS One 6:e21800. 10.1371/journal.pone.0021800

Symmons O, and Raj A. 2016. What's Luck Got to Do with It: Single Cells, Multiple Fates, and Biological Nondeterminism. Mol Cell 62:788-802. 10.1016/j.molcel.2016.05.023 
862 Tank PW, Carlson BM, and Connelly TG. 1976. A staging system for forelimb regeneration in

863 the axolotl, Ambystoma mexicanum. J Morphol 150:117-128. 10.1002/jmor.1051500106

864 The Gene Ontology Consortium. 2015. Gene Ontology Consortium: going forward. Nucleic

$865 \quad$ Acids Res 43:D1049-1056. 10.1093/nar/gku1179

866 Vogt G. 2015. Stochastic developmental variation, an epigenetic source of phenotypic diversity

867 with far-reaching biological consequences. J Biosci 40:159-204.

868 Voss SR, Palumbo A, Nagarajan R, Gardiner DM, Muneoka K, Stromberg AJ, and Athippozhy

869

870

871

872

873

874

875

876

877

878

879

880

881

882

883

884

885

886

887

888

889

890

891

892

893

894

895

896

897

898 AT. 2015a. Gene expression during the first 28 days of axolotl limb regeneration I: Experimental design and global analysis of gene expression. Regeneration 2:120-136. $10.1002 / \mathrm{reg} 2.37$

Voss SR, Woodcock MR, and Zambrano L. 2015b. A tale of two axolotls. BioScience 65:11341140.

Wang QM, and Wang L. 2012. An evolutionary view of plant tissue culture: somaclonal variation and selection. Plant Cell Rep 31:1535-1547. 10.1007/s00299-012-1281-5

Wilcoxon F. 1945. Individual Comparisons by Ranking Methods. Biometrics Bulletin 1:80-83. Doi $10.2307 / 3001968$

Wu C-H, Tsai M-H, Ho C-C, Chen C-Y, and Lee H-S. 2013. De novo transcriptome sequencing of axolotl blastema for identification of differentially expressed genes during limb regeneration. BMC Genomics 14:1. DOI: 10.1186/1471-2164-14-434

Wu CH, Huang TY, Chen BS, Chiou LL, and Lee HS. 2015. Long-duration muscle dedifferentiation during limb regeneration in axolotls. PLoS One 10:e0116068. 10.1371/journal.pone.0116068

Yamada Y, Haga H, and Yamada Y. 2014. Concise review: dedifferentiation meets cancer development: proof of concept for epigenetic cancer. Stem Cells Transl Med 3:11821187. 10.5966/sctm.2014-0090

Yanagida H, Gispan A, Kadouri N, Rozen S, Sharon M, Barkai N, and Tawfik DS. 2015. The Evolutionary Potential of Phenotypic Mutations. PLoS Genet 11:e1005445. 10.1371/journal.pgen.1005445

Zhu W, Kuo D, Nathanson J, Satoh A, Pao GM, Yeo GW, Bryant SV, Voss SR, Gardiner DM, and Hunter T. 2012a. Retrotransposon long interspersed nucleotide element-1 (LINE-1) is activated during salamander limb regeneration. Dev Growth Differ 54:673-685. 10.1111/j.1440-169X.2012.01368.x

Zhu W, Pao GM, Satoh A, Cummings G, Monaghan JR, Harkins TT, Bryant SV, Randal Voss S, Gardiner DM, and Hunter T. 2012b. Activation of germline-specific genes is required for limb regeneration in the Mexican axolotl. Dev Biol 370:42-51.

10.1016/j.ydbio.2012.07.021 


\section{Figure 1}

Temporal dynamics of the level and the heterogeneity in gene expression along axolotl forearm regenerative development.

Generalized changes in gene expression measures along axolotl forearm regeneration was inspected using data originally produced by Voss and coworkers and unrestricted MCW tests (see Material and Methods for further details). Gene expression bias indexes (GEBls) measure generalized biases for transcript abundance $\mathrm{CV}$ and mean (cVGEBls and mGEBls respectively) when comparing data for each post-amputation timepoint (TX) and the day of the amputation (T0), TX vs TO comparisons. Positive GEBIs represent timepoints for which gene expression measures tend to be higher post-amputationally than in the day of amputation ( $T X>T 0$ ), whereas negative GEBIs represent cases for which gene expression measures tend to be lower post-amputationally than in the day of amputation $(T X<T 0)$. Simulated GEBIs were obtained after randomly rearranging gene expression measures for all probesets within TX vs T0 comparison 10,000 times with no restriction. The distribution of simulated GEBIs is summarized using minimums, 5th and 95th percentiles, and maximums. Observed GEBls were considered significant if they outlied the area of the graph defined by 5th and 95th percentiles $(P<0.05)$. Grey boxes delimit axolotl forearm regeneration stages defined by morphological changes according to (Voss et al. 2015) . 


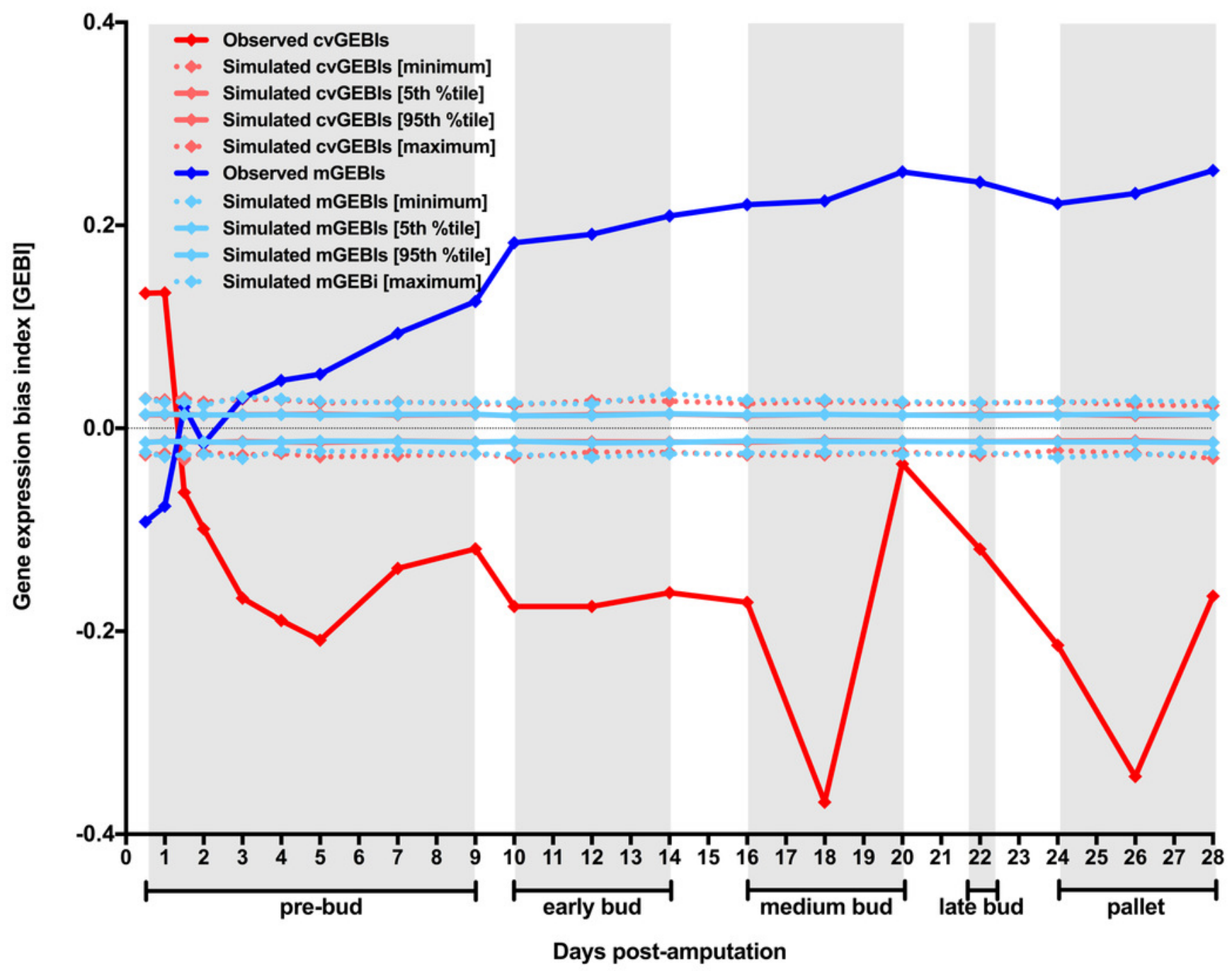




\section{Figure 2}

Contribution of chance, factors acting on the transcriptome as a whole, and the variation in gene expression level to gene expression heterogeneity dynamics along axolotl forearm regenerative development.

MCW tests using transcript abundance CV for each TX vs T0 comparison were performed by randomly rearranging transcript abundance CV with no restriction (unrestricted), restricted by timepoint (timepoint-restricted), or restricted within probeset bins defined according to their transcript abundance mean values (expression-restricted). Two alternative expressionrestricted MCW test designs performed independently are indicated with different superscripts (see Material and Methods for further description). MCW tests were repeated 10,000 times, and simulated cVGEBIs with closer values to observed cvGEBIs are represented in panel A. (B) The fraction of observed cVGEBI dynamics that could be explained by chance, or factors acting on the transcriptome as a whole, or the variation in gene expression level were calculated as the proportional cumulative area under the curve ( $\left.\mathrm{PC}_{\mathrm{AUC}}\right)$.
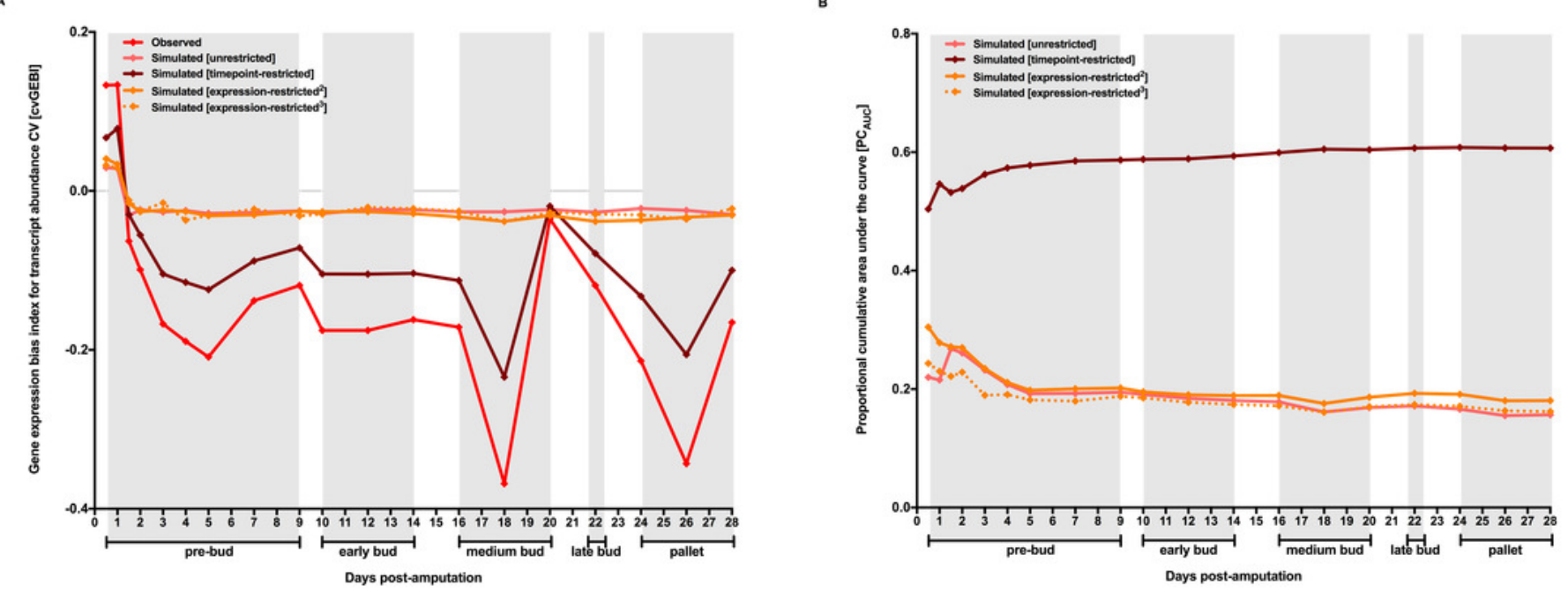


\section{Figure 3}

Temporal dynamics of the level and the heterogeneity in gene expression along axolotl forearm regenerative development for groups of genes defined by their functionality.

The Gene Ontology Consortium database was used to retrieved genes belonging to "mitotic cell cycle process" (GO:1903047) and "chromatin organization" (GO:0006325) GO terms (The Gene Ontology Consortium 2015). The temporal dynamics of the level and heterogeneity in gene expression for probesets associated with these two groups of genes with regard to the whole transcriptome were inspected using functionally-restricted MCW tests. CVGEBI and mGEBI for each group of probesets and TX vs T0 comparison were calculated before and after randomly rearranging functional subset tags for the whole transcriptome 10.000 times. The distribution of simulated GEBIs is summarized using minimums, 5th and 95th percentiles, and maximums. Observed GEBls were considered significant if they outlied the area of the graph defined by 5th and 95th percentiles $(P<0.05)$. Grey boxes delimit axolotl forearm regeneration stages defined by morphological changes according to (Voss et al. 2015) .
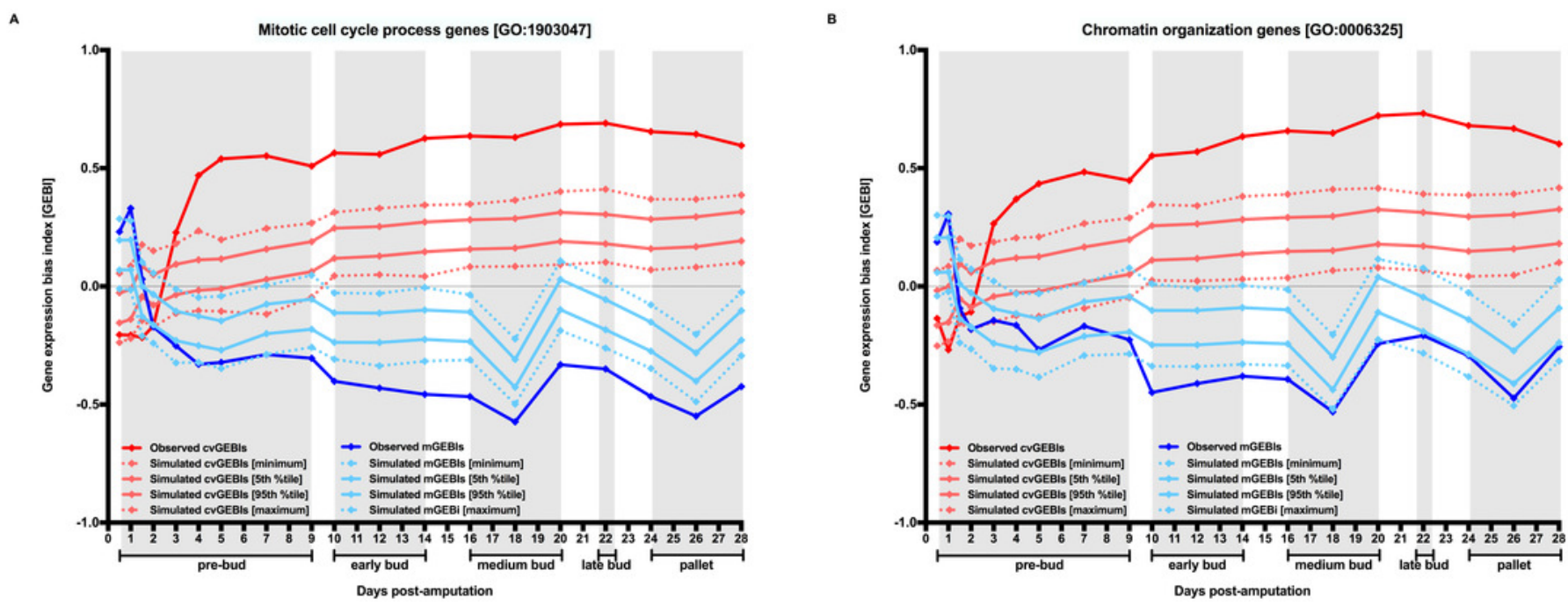


\section{Table $\mathbf{1}$ (on next page)}

Gene Ontology (GO) enrichment for genes prioritized with regard to their change in gene expression heterogeneity between 1 and 1.5 days after axolotl forearm amputation.

Probesets in Voss et al. dataset were prioritized upon their transcript abundance CV

difference between 1 and 1.5 post-amputation timepoints, and randomly rearrange 10 times.

GOrilla and REVIGO were used to perform gene ontology (GO) enrichment analyses on

prioritized and random lists. The GO term enrichment found using random lists supported by

the largest number of genes was used as threshold to narrow down observed GO

enrichments with potential biological significance. See Materials and Methods for further

details, and Table S3 for the complete list of GO term enrichments found for prioritized and

random lists. Bold names represent genes found supporting both $\mathrm{GO}$ term enrichments. 
1

\begin{tabular}{|c|c|c|c|c|}
\hline GO Term & Description & $P$ value & Enrichment & Genes \\
\hline GO:1903047 & mitotic cell cycle process & 0.00014 & 1.54 & $\begin{array}{l}{[S E P T 7, \text { KLHL42, CDK5RAP2, }} \\
\text { CHAMP1, PAPD5, GSPT1, ORC4, } \\
\text { CD2AP, SPDL1, MUS81, GSG2, KIF2A, } \\
\text { MAP4, PIBF1, CNOT11, PPM1D, } \\
\text { USP16, SPAST, ESPL1, ID2, PSMB3, } \\
\text { CDCA5, EIF4E, PSMA5, PBRM1, SMC5, } \\
\text { EML4, PSMB6, CHMP5, PSMC5, } \\
\text { MAP10, RAD17, CACUL1, PSMC1, } \\
\text { NOLC1, FBXW11, CEP152, DYNLT3, } \\
\text { PSMD7, INTS3, LMNA, SMC4, PSMD2, } \\
\text { PSMD12, CEP250, PSMD11, CUL5, } \\
\text { MSH2, RAN, PSMD13, TAF10, FBXL15, } \\
\text { NIPBL, TUBA1A, MYH10, MIS12, } \\
\text { CCNG2, CEP70, MAP9, CCNG1, VPS4B, } \\
\text { MYBL2, TOP2B, TFDP2, TOP2A, CLTA, } \\
\text { TUBB4B, HMGA2, RPS6KB1, CDK6, } \\
\text { DYNC1H1, RBBP8, DYNC1I2, BABAM1, } \\
\text { RAB11A, CDK7, DNM2, OFD1, STAG2, } \\
\text { CEP192, NDC80, NDC1, NR3C1, } \\
\text { HELLS, RUVBL1, CSNK1A1, CCNB1, } \\
\text { KIF23, NUDC, CDCA2, HMMR, } \\
\text { NUP153, ZNF207] }\end{array}$ \\
\hline GO:0006325 & chromatin organization & 0.00025 & 1.61 & $\begin{array}{l}\text { [ZMYND8, SMARCAD1, VPS72, GSG2, } \\
\text { ARID5B, TLK1, BAHD1, NCOR1, } \\
\text { PRMT6, KDM5B, SUPT5H, SIRT5, } \\
\text { MTA1, USP16, BPTF, DOT1L, KAT2A, } \\
\text { BRD8, CHD2, USP7, UTP3, WHSC1, } \\
\text { PBRM1, TTF1, PER1, TET3, SCMH1, } \\
\text { MTA2, NUCKS1, CREBBP, MORF4L1, } \\
\text { CENPP, WDR82, TAF10, MTF2, TAF5, } \\
\text { SUPT16H, INO8OC, SUPT7L, DNAJC2, } \\
\text { HIRA, NFE2, SMARCE1, BRD2, ATRX, } \\
\text { RNF2, ATXN7L3, BAZ1A, BAZ1B, } \\
\text { HMGA2, PHF20, SMARCC1, PRDM2, } \\
\text { BABAM1, SRPK1, OGT, ARID4A, TET2, } \\
\text { HIRIP3, CBX3, SMARCA2, NR3C1, } \\
\text { HELLS, RUVBL1, SETD2, TLK2, } \\
\text { CCNB1, RBM14, KAT6B, PRKCD, } \\
\text { ZNF462, ELP3] }\end{array}$ \\
\hline
\end{tabular}




\section{Figure 4}

Generalized reduction in gene expression heterogeneity upon cell dedifferentiation might reduce genetic capacitance.

Cartoon symbolizing how genetic variation can become phenotypically relevant associated to the chromatin relaxation-dependent reduction in gene expression heterogeneity upon cell dedifferentiation. Blue shades represent the variation in gene expression for a particular gene in a population of cells before and after dedifferentiation. Pre-existing or new genetic mutations might be phenotypically more distinguishable upon cell dedifferentiation because of the generalized reduction in gene expression heterogeneity that cause a narrowing of the spectrum of stochastic phenotypes.

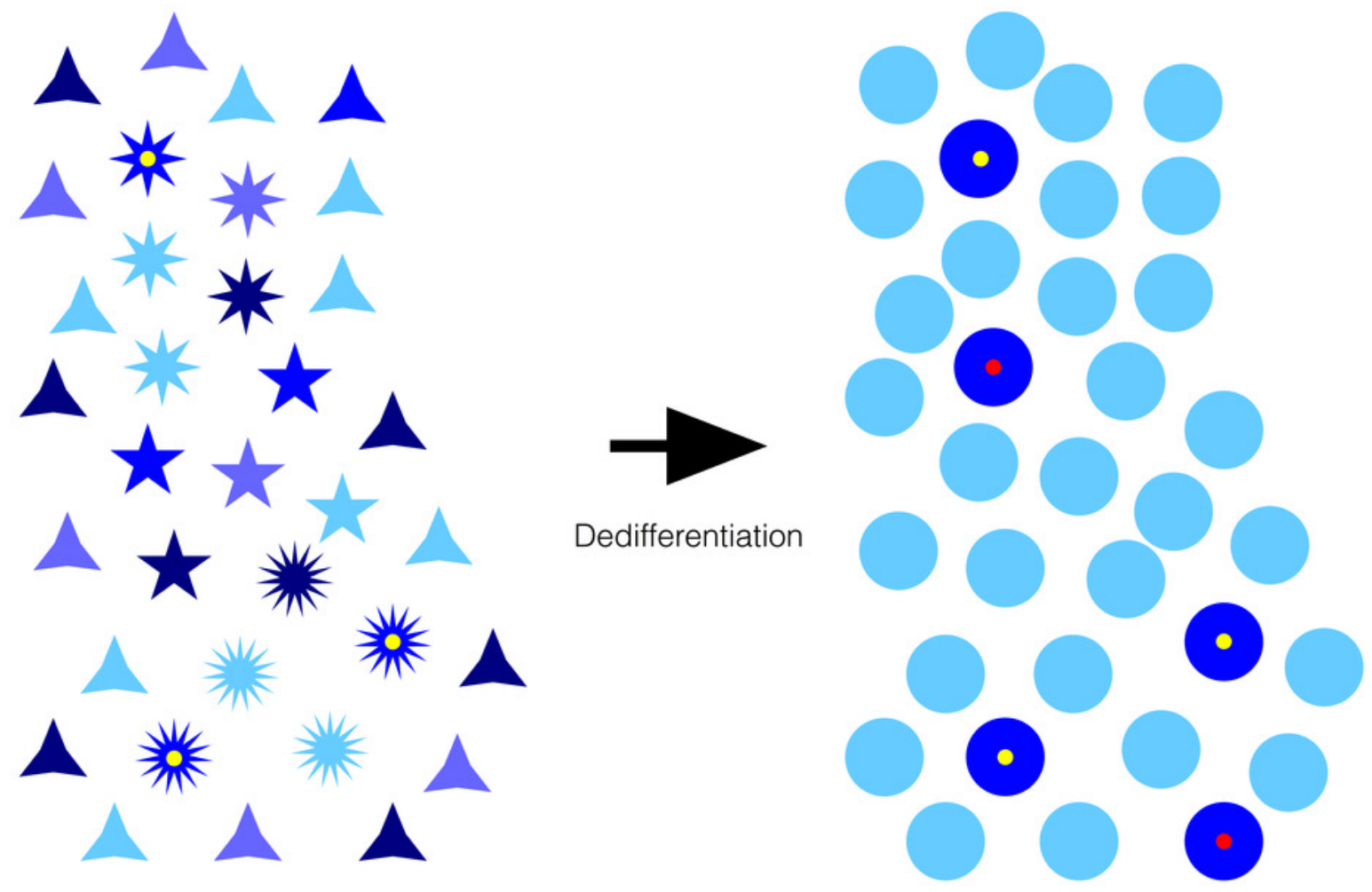

